\title{
Optimization of High Power Diode Laser Module End Pumped Nd:YAG
}

\author{
Salah H. Elnaby, Adel M. Elnozhy, Arafa K. Kassem, and Sameh M. \\ Helaly
}

National Institute of Laser Ennhanced Science, Cairo University, Egypt

In the present paper a Nd:YAG crystal pumped with a high power fiber-coupled diode laser module at $808 \mathrm{~nm}$ is optimized by using the simulation of the LASCAD software. The module is used to investigate the optimum cavity design parameters. In the present paper was optimized the cavity design parameters by controlling the cavity length, the beam overlap parameter, the Nd:YAG rod dimension, and the output coupler reflectvity. The practical example of a Nd:YAG laser pumped by a $7.8 \mathrm{~W}$ fiber-coupled laser diode module is considered to confirm the suggestes physical analysis. The cavity optimum configuration was cavity length $65 \mathrm{~mm}, \mathrm{Nd}: Y A G$ rod dimension $\phi 3 \mathrm{mmx} 10 \mathrm{~mm}$ with $1 \%$ doping concentration (1at.\%), and output coupler reflectivity $85 \%$. The pump waist spot size $w_{g}$ equal to the beam waist spot size $w_{g}$. The final optical to optical efficiency is $39 \%$.

\section{Introduction:}

The need for $1064 \mathrm{~nm}$ high power laser system, which has the specifications of low power consumption and lowest pumping threshold, high slope efficiency, compact design, and frequency stability, is very important. In general, stable high power continuous laser output was hardly obtained because of the increase of thermal loading. The challenge is to design a more stable continuous high power laser system with reduction in thermal loading that produces high conversion slop efficiency. Diode pumped Nd:YAG laser system is found to be the most motivate system achieving all previous benefitiale features, further producing a reliable system with a lower cost.

\section{Optimization Cavity Length:}

The effects an the optical system which is denoted by the thermal lens of the laser crystal which is an important parameter for optimizing the laser system and performing the high power laser operation. When a fiber-coupled diode laser pumped $\mathrm{Nd}$ :YAG crystal in CW mode of operation, the resonator experienced a strong thermal lensing effect with Nd:YAG. The thermally induced focusing lens derives the laser resonator out of the stable regime. In the absence of laser action, the thermal loading on the medium is strong because of the strong heat absorption within the material [1]. Therefore, the 
thermally induced focusing lens of the material is stronger in non-lasing operation. However, the main concerning point is to determine the pump-induced thermal lens of the Nd:YAG laser medium lasing condition by using a fiber-coupled diode laser pump. After thermally induced focusing lens was identified, the resonator would be redesigned to achieve a stable laser oscillation.

The effective focal length for entire Nd:YAG crystal is given by [2].

$$
f=\frac{\pi K_{c} w_{g}^{2}}{P_{p h}(d n / d T)}\left(\frac{1}{1-\exp (-\alpha l)}\right)
$$

where $P_{p h}$ is the fraction of the pumped power that results in heating which is given by $P_{p h}=\left(1-\frac{\lambda_{P}}{\lambda_{L}}\right) P_{P}$. where $\lambda_{L}$ is the emitted laser wavelength (1064nm), and $\lambda_{P}$ is the pumped diode wavelength $(808 \mathrm{~nm}), K_{c}$ is the thermal conductivity and equal $0.14 \mathrm{~W} \mathrm{~cm}^{-1} \mathrm{~K}^{-1}, d n / d T$ is the temperature coefficient of the refractive index and equal $7.3 \times 10^{-6} \mathrm{~K}^{-1}, \alpha$ is the absorption coefficient of the $\mathrm{Nd}$ :YAG crystal which depends on the $\mathrm{Nd}^{+3}$ doping percentage (in case of 1.0 at $\% \alpha=6 \mathrm{~cm}^{-1}$ ), $w_{g}$ is the spot size of the pump beam waist (in the present study $w_{g}=150 \mu \mathrm{m}$ ), and $l$ is the rod length (real dimension was $10 \mathrm{~mm}$ ).

Then $P_{p h}$ is found to be equal to $\left(0.24 \mathrm{x} P_{P}\right)$. For input pumped power of $7.8 \mathrm{~W}$, $P_{p h}$ calculated to be equal $1.872 \mathrm{~W}$ which is a close result to the previous performance modeling of end pumped Nd:YAG. And the effective focal length for the entire crystal calculated to be equal to $7.2 \mathrm{~cm}$.

The importance of these results is that, the effective focal length of the required pumping rod is actually comparable to the resonator length. To verify this condition different cavity lengths were simulated with the same rod of dimension $3 \times 10 \mathrm{~mm}$ and with the same output reflectivity of $85 \%$ at $1064 \mathrm{~nm}$. Considering the previous worst case of the transfer efficiency $\eta_{T}=0.80$, this will be simulated using LASCAD software package. The simulation results are shown in Fig.(1). 


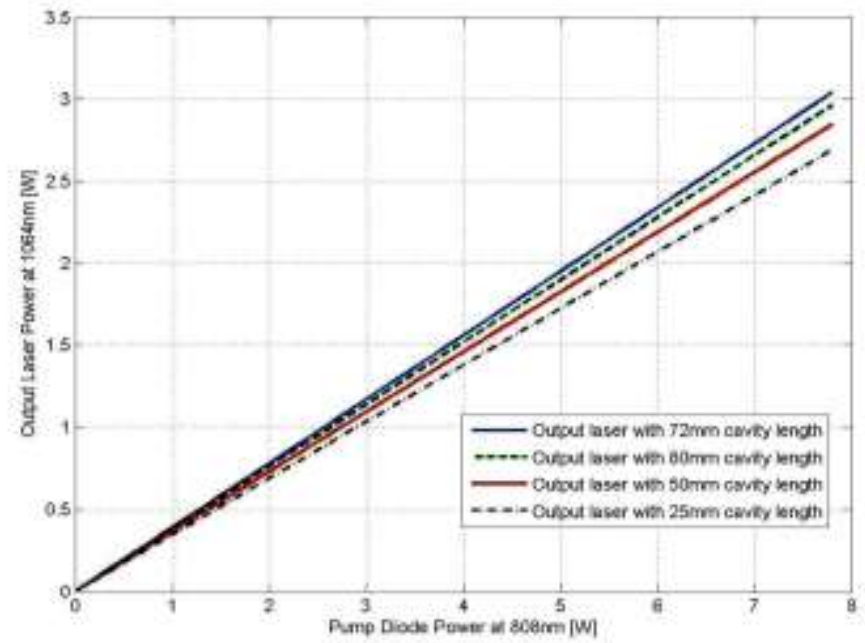

Fig.(1): The simulation results of changing cavity length on the output laser power at $1064 \mathrm{~nm}$

From Fig.1. we can notice that the maximum output power is $3.042 \mathrm{~W}$ at the cavity length of $72 \mathrm{~mm}$ and producing an optical efficiency of $39 \%$ [3].

\section{Optimizisation Nd:YAG Crystal Length:}

The effect of changing crystal length on the extracted laser output is investigated. The diode pumped Nd:YAG crystal with different crystal lengths was simulated. Fig.(2). shows the laser output power at $1064 \mathrm{~nm}$ versus different crystal lengths $(10,15,20$, $30 \mathrm{~mm}$ ). It illustrates the independency of the laser output on changing rod length. Moreover, increasing the length of the gain medium will produce insignificant changing in the extracting power. This change is inversely proportional to the rod length [3].

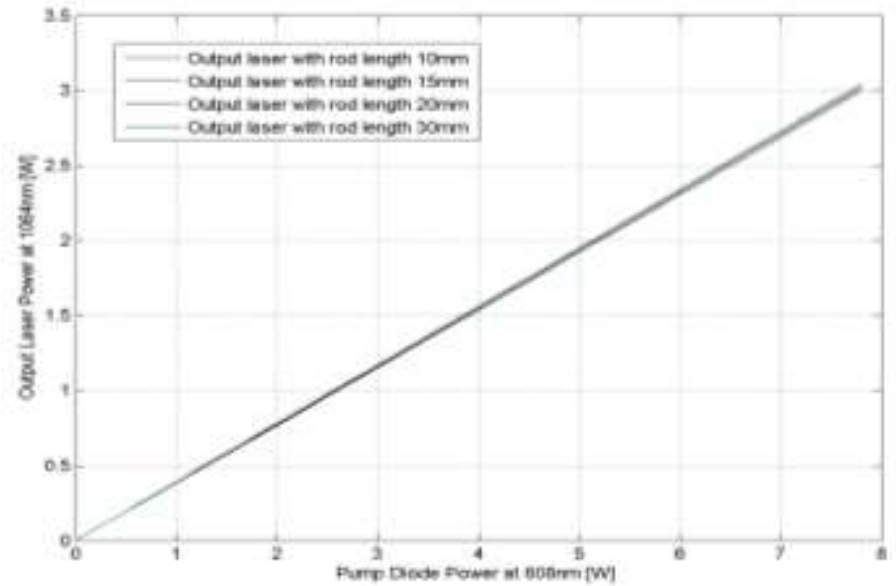

Fig.(2). The simulation results of changing the rod length on the output laser power at $1064 \mathrm{~nm}$ 
The total absorption efficiency in case of end pumping Nd:YAG crystal will be arround $72 \%$, so using $10 \mathrm{~mm}$ crystal length of Nd:YAG crystal provide output laser power at $1064 \mathrm{~nm}$ slightly higher than crystal of $40 \mathrm{~mm}$ in length as shown in Fig.(2).

\section{Optimizat Pumped Beam Waist:}

As mentioned before the resonator length considered to be an important factor in the end pumping design issues. It depends on the square input beam radius of the waist spot size and independent on the rod length. So the only parameter that can optimize the cavity design is the input beam waist which maximizes the performance of the output laser at $1064 \mathrm{~nm}$ at the given pumped power [4]. In end pumping solid state laser controlling $w_{g}$ achieved the benefits of the $\mathrm{TEM}_{00}$ with extracting maximum possible output power. The ratio between $w_{B}$ and $w_{g}$ affects the diode pumped solid state laser system performance output $\eta_{B}=\frac{2 w_{B}^{2}}{w_{g}^{2}+w_{B}^{2}}$ for $w_{g}>w_{B}$ and $\eta_{B}=1$ for $w_{g} \leq w_{B}$, this will be simulated using LASCAD software package.

By simulating a diode end pumped Nd:YAG with variation in the pump waist spot size $w_{g}$ at the same crystal dimension $3 \times 10 \mathrm{~mm}$ and the output coupler of $85 \%$ reflectivity at $1064 \mathrm{~nm}$. Fig.(3). shows the simulated results of the output laser at $1064 \mathrm{~nm}$ versus the input pump laser at $808 \mathrm{~nm}$ and illustrates the effect of changing $w_{g}$ size on the output laser performance from Fig.(3). It is noticed that the ratio between $w_{B}$ and $w_{g}$ affects the system output performance, as the maximum output laser power is $3.042 \mathrm{~W}$ can be extracted when this ratio exactly equal to $1.0\left(w_{B} \approx w_{g} \approx 150 \mu \mathrm{m}\right)$.

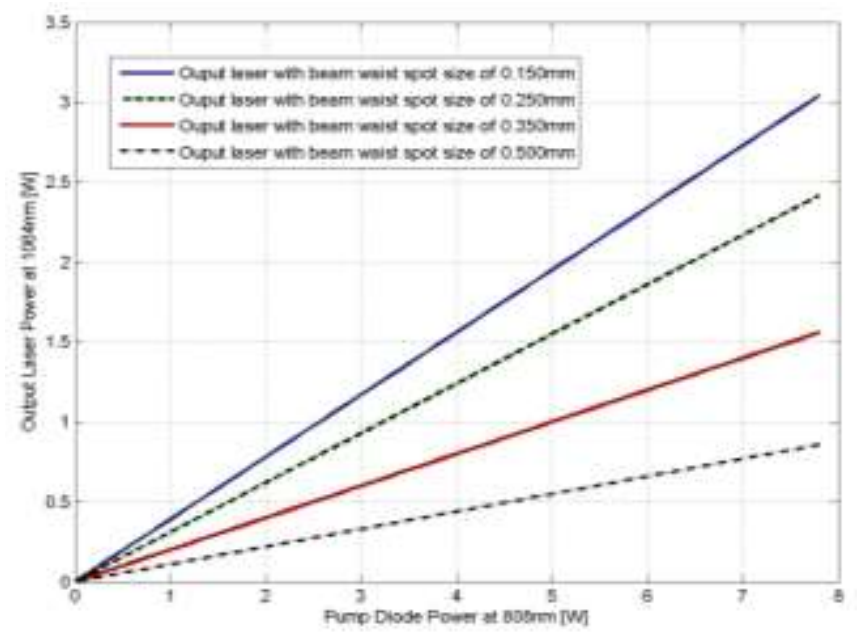

Fig.(3): The simulated results of changing beam waist spot size on the output laser power at $1064 \mathrm{~nm}$ 
Figure (4). shows the LASCAD mode profile menu illustrating the beam overlap between the pump beam spot size and the beam waist spot size. As this ratio smaller than 1.0, the extracted power will be much less than the expected (case of $\eta_{B}=1$ ), this effect is shown at a given beam waist spot size $w_{g}$ is 250,350 , and $500 \mu \mathrm{m}$ [3].
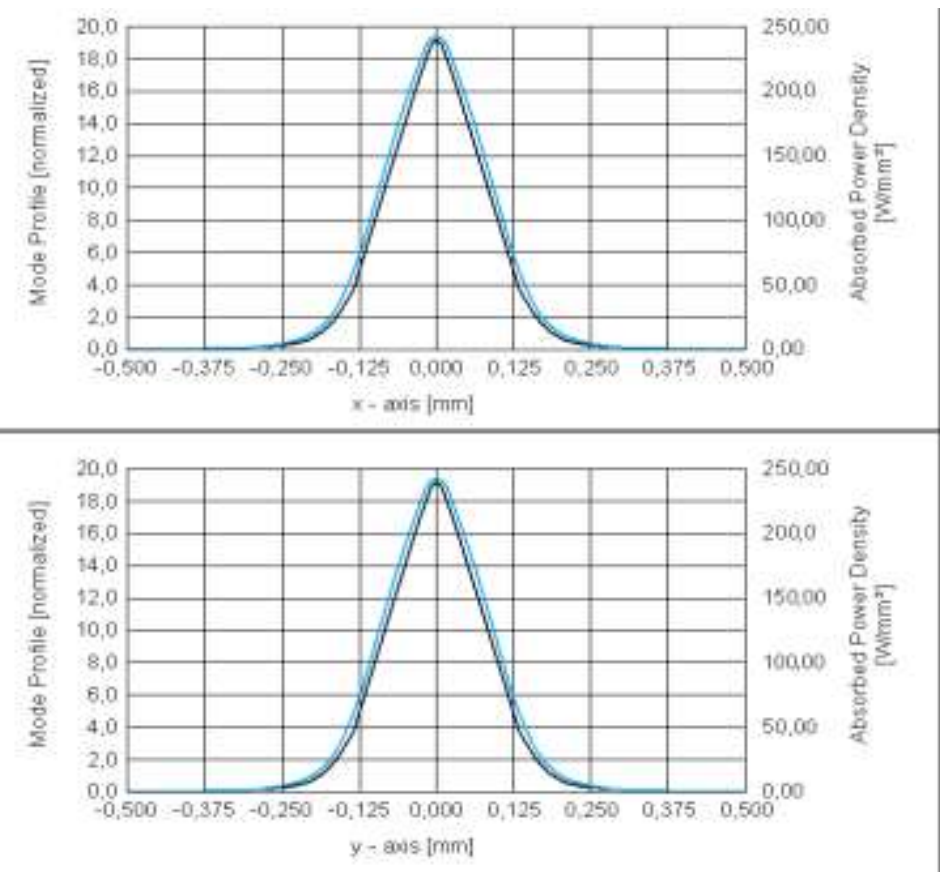

Fig.(4): LASCAD simulation of the overlap between bump beam spot size and beam waist spot size

\section{Optimizat Output Coupler Reflectivity:}

A low transmission output coupler leads to a low laser thershold, but also possibly to poor laser efficiency if the losses due to the output coupler do not dominate over other parasitic losses in the laser cavity $\eta_{c}=\frac{T}{(L+T)}$. So we must optimize the output coupler reflectivity by simulate the effect of changing output coupler reflectivity on the extracted laser output is investigated. A set of output couplers with different percentage of reflectivity $(85,90$, and $95 \%)$ at $1064 \mathrm{~nm}$ are used to facilitate the studying of output coupler effect on the extracted laser power. 


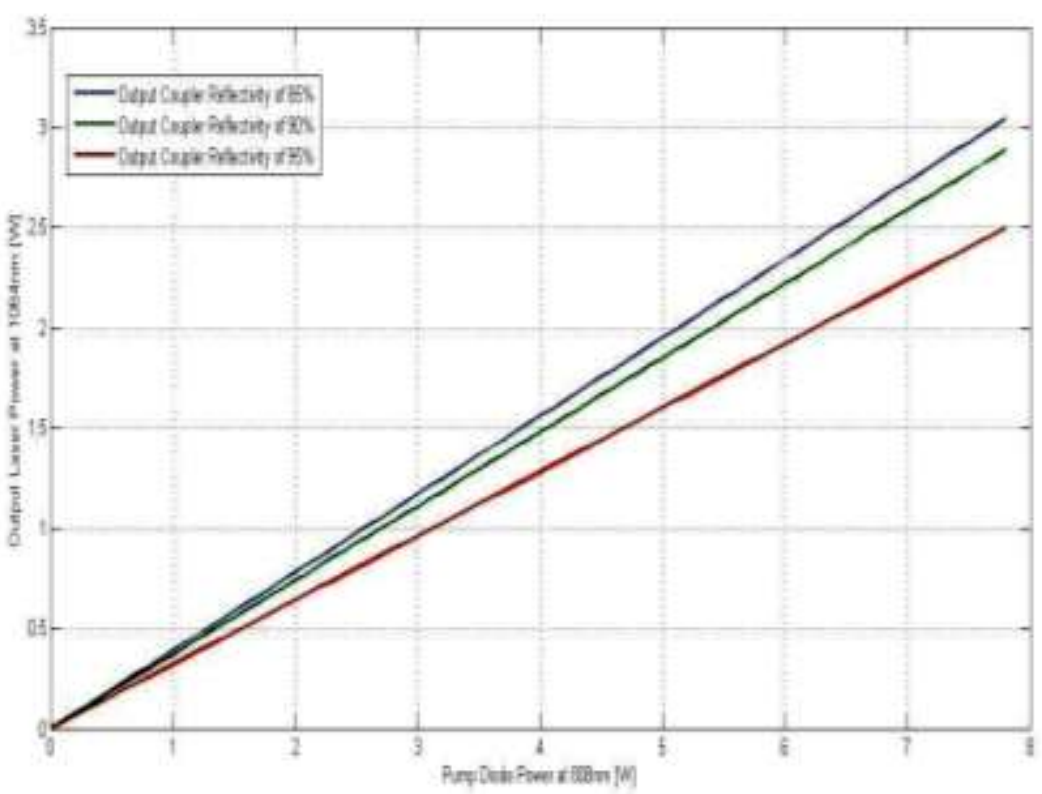

Fig.(5): The simulated results of changing output coupler reflectivity on the output laser power at $1064 \mathrm{~nm}$

Figure (5): shows the output laser power at $1064 \mathrm{~nm}$ verse output coupler reflectivity, at a pumped power of $7.8 \mathrm{~W}$. From the Fig.2.15 it can be notice that the maximum extracted laser power at $1064 \mathrm{~nm}$ is $3.042 \mathrm{~W}$ with an output coupler of $85 \%$ reflectivity.

\section{Stability Simulation of the Laser Cavity:}

A simple geometric statement describes the regions of stability: A cavity is stable if the line segments between the mirrors and their centers of curvature overlap, but one does not lie entirely within the other. From the previous simulations, which studied different cavity sizes, it is important to discuss the Nd:YAG resonator stability for each case individually using the LASCAD software. The plano-concave cavity resonator was used. A cavity composed of two mirrors, the input mirror is a flat mirror high reflection coated at $1064 \mathrm{~nm}$ and high transmission coated at $808 \mathrm{~nm}$, and the output coupler is a concave mirror with radius of curvature of $100 \mathrm{~mm}$ HR at $808 \mathrm{~nm}$ with $15 \%$ transmission factor at $1064 \mathrm{~nm}$. 


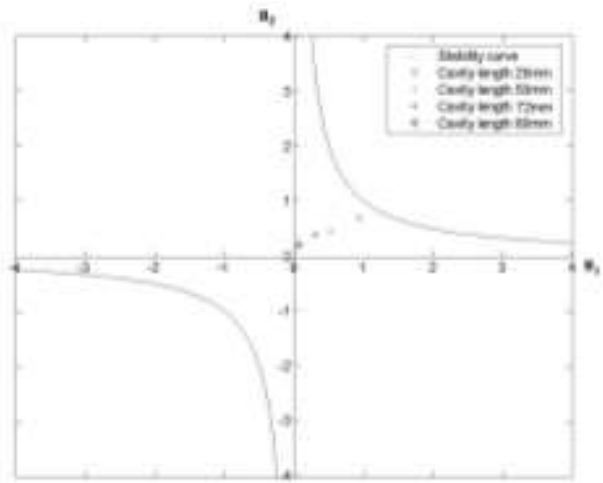

a)

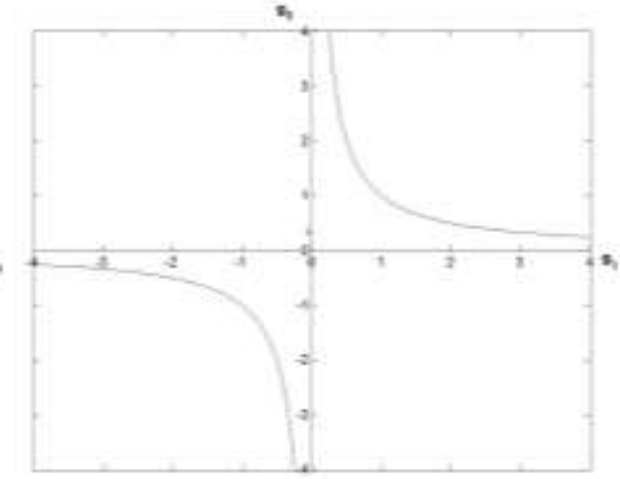

b)

Fig.(6): The simulated stability of the Nd:YAG designed cavity with lengths a) $25,50,72$ and $80 \mathrm{~mm}$. b) above $80 \mathrm{~mm}$.

Figure (6): shows the stability condition realization of the Nd:YAG resonator cavity various designed lengths $25,50,72$, and $80 \mathrm{~mm}$. We can notice that at cavity lengths $25,50,72$, and $80 \mathrm{~mm}$ the cavity is stable and the unstability of the $\mathrm{Nd}$ :YAG resonator cavity at length above $80 \mathrm{~mm}$.

\section{Simulation of the Absorbed Power Density in Nd:YAG Crystal:}

The absorbed power density along and at the front pumped face of the Nd:YAG rod can be simulated for the two different rod lengths $10 \mathrm{~mm}$ and $20 \mathrm{~mm}$ by using the LASCAD software as shown in Fig.7. [3].

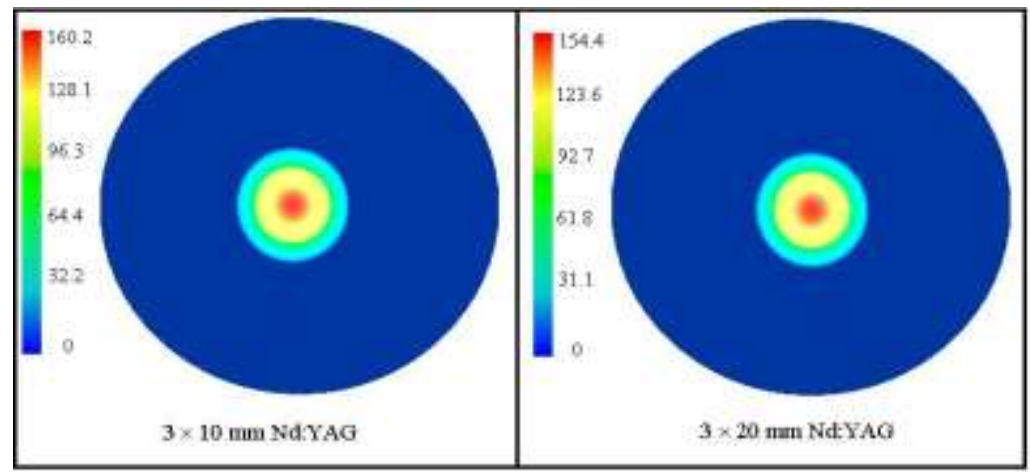

Fig.(7): LASCAD simulation of the absorbed power density $\left(\mathrm{W} / \mathrm{mm}^{3}\right)$ in $\mathrm{Nd}$ :YAG rod a) Nd:YAG rod of dimension 3x20mm; b) Nd:YAG rod of dimension 3x10mm.

From Fig.7. it can be noticed that the absorbed power density $\left(\mathrm{W} / \mathrm{mm}^{3}\right)$ along the Nd:YAG rod independent on the Nd:YAG rod length.

\section{Simulation of Heat Distribution in Nd:YAG crystal:}


It is important to simulate the temperature gradient inside the Nd:YAG crystal to avoid thermally induced fracture and evaluate the maximum pumped power. Fig.8. shows the simulated results of two rods of different dimensions namely $3 \times 10 \mathrm{~mm}$ and $3 \times 20 \mathrm{~mm}$.

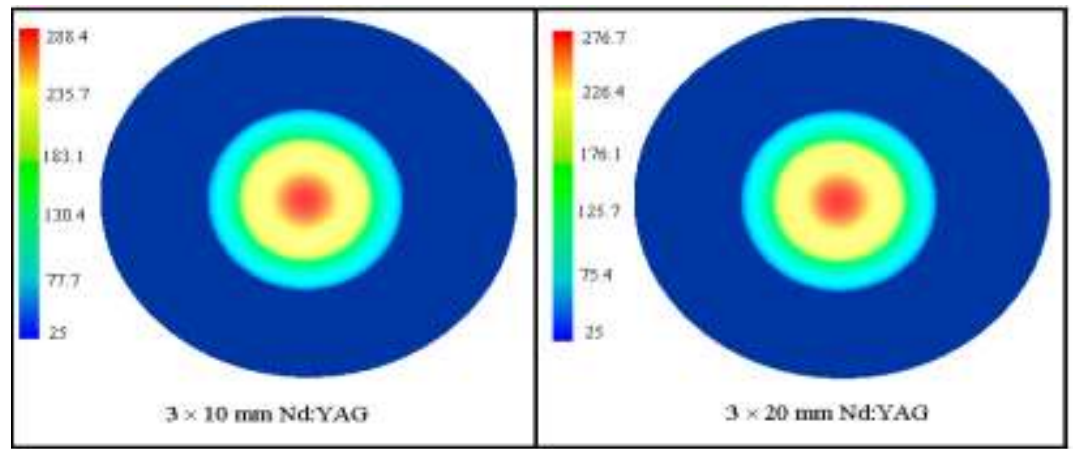

Fig.(8): LASCAD simulation of the Temperature in two different Nd:YAG rod lengths.

The results shows the thermal gradient inside the Nd:YAG crystal, and show a central maximum temperature of $288^{\circ} \mathrm{C}$ and $276^{\circ} \mathrm{C}$ for the first and the second rod respectively with pump diode power $7.8 \mathrm{~W}$ by using LASCAD. The simulation was deliberated to confirm that the longer crystal is useful in heat removal, but it increases the intracavity absorption losses as shown previously in Fig.(2).

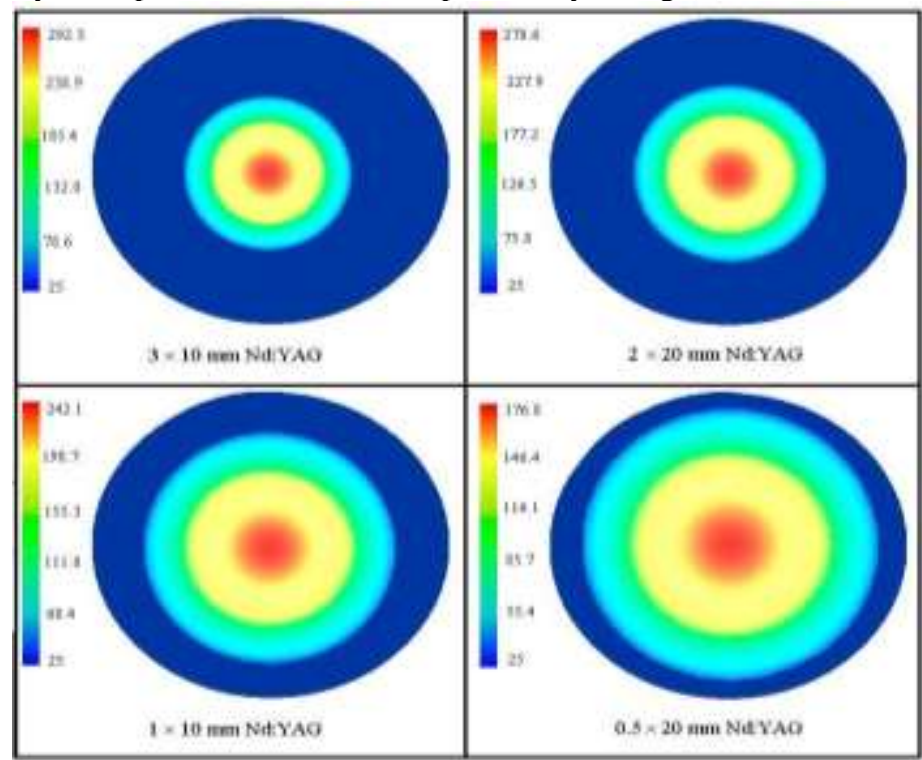

Fig.(9): LASCAD simulation of the temperature distribution in different Nd:YAG rod diameters.

Also the temperature gradient is affected by the radial change of the crystal diameter, so we simulate the results of different rods diameter. it can be seen that as the 
crystal diameter became smaller; minimum storage temperature case is obtained. This is because the deposited heat can be more effectively removed radially from the crystal. Then the fracture limit can be increased as the crystal diameter became smaller. So the more pumping can be tolerated with smaller radial diameter [5].

\section{Conclusion:}

The cavity design parameters were optimized by controlling the cavity length, the beam overlap parameter, the Nd:YAG rod dimension, and the output coupler reflectvity. The practical example of a Nd:YAG laser pumped by a $7.8 \mathrm{~W}$ fiber-coupled laser diode module is considered to confirm suggested physical analysis. The cavity opimum configuration was cavity length $65 \mathrm{~mm}, \mathrm{Nd}$ :YAG rod dimension $\emptyset 3 \mathrm{mmx} 10 \mathrm{~mm}$ with $1 \%$ doping concentration (1at.\%), and output coupler reflectivity $85 \%$. The pump waist spot size $w_{g}$ equal to the beam waist spot size $w_{g}$. The final optical to optical efficiency is $39 \%$. Also the stability of the system must be checked and the heat distribution inside the Nd:YAG laser rod should be understand.

\section{References:}

1. YANG Ji-min, Liu Jie, Je Jing-liang, Journal of Opto Electronics Letters, 1, 44, (2005).

2. Walter Koecner, and Michael Bass, "Solid-State Laser Engineering", fourth edition, Springer, (1999).

3. LASCAD 3.3.5 Software Manual, September 14, (2007).

4. J. C. Ricklin, W. B. Miller, and L. C. Andrews, Journal of Applied Optics, 34, 7059, (1995).

5. Y. F. Chen, T. S. Liao, C.F. Kao, T.M. Huang, K. H. Lin, S. C. Wang, IEEE, Journal of Quantum Electronics, 32, 2020, (1996). 